\title{
A New Approximate Solution for Diffusional Growth and Dissolution of Spherical Precipitates
}

\author{
Akira Seki ${ }^{1, *}$ and Kotarou Hayashi ${ }^{2}$ \\ ${ }^{1}$ Nippon Steel Technology Co., Ltd., Amagasaki 660-0891, Japan \\ ${ }^{2}$ Nippon Steel Corporation, Amagasaki 660-0891, Japan
}

A new approximate solution for diffusional growth and dissolution of spherical precipitates has been proposed. The exact analytical solutions of the time-dependent diffusion equation with the flux balance at the interface is often difficult to obtain. The approximate solutions are useful even when the exact solutions are obtainable. The present approximation is the modified version of the conventional linearized gradient approximations, which are tractable for the spherical precipitates growth but does not agree accurately with the exact solution. It is shown that the present model is better than the conventional approximations. The present model is also applicable to the spherical precipitates dissolution, which has no exact solution. The present model gives an ordinary differential equation for dissolution, which can be solved numerically. [doi:10.2320/matertrans.MT-M2020310]

(Received September 29, 2020; Accepted November 11, 2020; Published December 11, 2020)

Keywords: spherical precipitate, diffusion equation, diffusional growth, diffusional dissolution, linearized gradient approximation

\section{Introduction}

The diffusion-controlled kinetics of growth and dissolution of the precipitates in the alloys are described by the analytic solutions of mathematical models for the diffusional phase transformation. The mathematical models are the diffusion equations with a moving boundary. Analytical solutions are as useful as numerical solutions to understand the growth and dissolution kinetics. Exact analytical solutions are often difficult to obtain. The approximate solutions are useful even when the exact solutions are obtainable, because they are often rather complicated to use. Therefore, several approximate analyses are proposed to simplify the mathematics of the models. The present study is limited to the spherical precipitates.

In the present study the analytical solutions of the timedependent diffusion equation in spherical coordinate system with the flux balance condition at the interface are considered. ${ }^{1,2)}$ For growth the exact analytical solution ${ }^{2,3)}$ is available. The approximations used are the invariant field ${ }^{1,4)}$ (Laplace), the invariant-size ${ }^{1)}$ (stationary interface) and the linearized gradient approximations ${ }^{5,6)}$ for growth. The polynomial approximation, ${ }^{7)}$ which includes the linearized gradient approximation, is also proposed. The linearized gradient approximation is not a good approximation for growth in the spherical system, while in one dimensional system this approximation is a good approximation. For dissolution there is no exact solution available. ${ }^{1)}$ In addition the linearized gradient approximation in the spherical system is not convenient to use practically as the analytical form is complex. ${ }^{1)}$ Thus, the invariant field ${ }^{1)}$ and invariant size ${ }^{8)}$ approximations can only be used for dissolution, although they deviate from the exact solution at large supersaturation.

The purpose of this study is to propose a new approximation for the growth and dissolution of the spherical precipitates. The present approximation is the modification of the conventional linearized gradient approximation. The present approximate solution is much closer to the exact

*Corresponding author, E-mail: seki.akira.s5m@nstec.nipponsteel.com solution and easier to use than the conventional linearized gradient approximate solution.

The dissolution process is of practical importance as well as the growth process because it is often necessary to know the appropriate heat treatment required for homogenization of alloys. It is shown that the present approximation also can be used for the dissolution of spherical precipitates, where no exact solution is available.

\section{Analytical Solutions of the Diffusion Equation with a Moving Boundary}

The diffusional growth or dissolution of an isolated spherical precipitate in an infinite matrix is described by the radial diffusion equation for a constant diffusion coefficient as follows.

$$
\frac{\partial c}{\partial t}=\frac{D}{r^{2}} \frac{\partial}{\partial r}\left(r^{2} \frac{\partial c}{\partial r}\right)
$$

where $D$ is the volume diffusion coefficient in the matrix, $c=c(r, t)$ is the concentration field of the solute in the matrix surrounding the precipitate. The boundary and initial conditions for the growth and dissolution of the precipitates are following.

$$
\begin{gathered}
c(R, t)=c^{m}, \quad 0<t, \\
c(\infty, t)=c^{\infty}, \quad 0<t, \\
c(r, 0)=c^{\infty}, \quad 0<r, \\
R(t=0)=0, \quad \text { for growth, } \\
R(t=0)=R_{0}>0, \quad \text { for dissolution, }
\end{gathered}
$$

where, $R$ is the radius of the precipitates, $r=R$ at the precipitate/matrix interface, $c^{m}$ is the solute concentration in the matrix at the precipitate/matrix interface, and $c^{\infty}$ is the far-field solute concentration of the alloy. The schematic concentration fields for the growth and the dissolution of spherical precipitates are shown in Fig. 1. The field shown in the figure is for a solute-rich precipitate. In the following, solute-rich precipitates are considered but it is easy to extend the present analyses to solute-poor precipitates. 
(a)

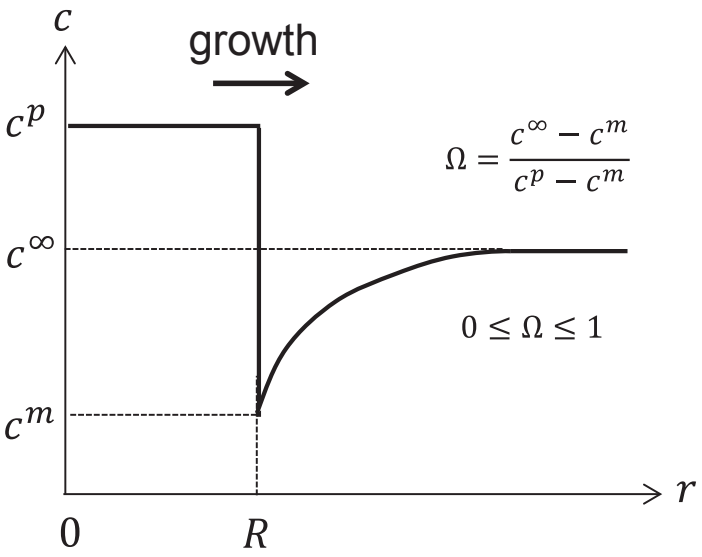

(b)

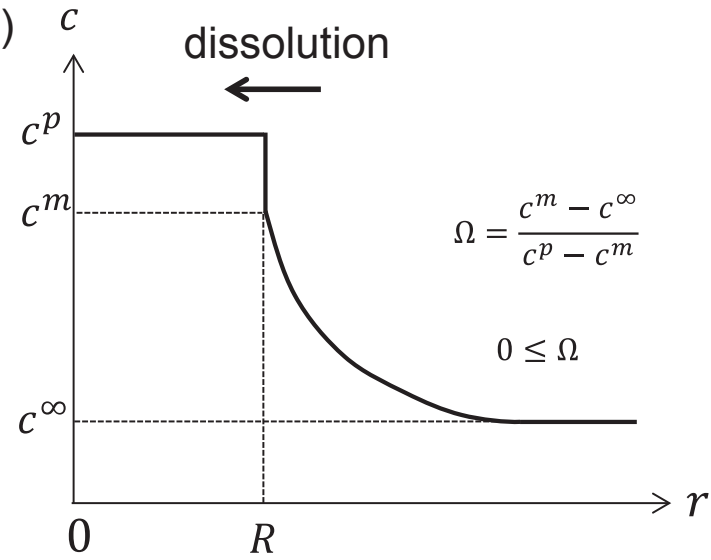

Fig. 1 Schematic of concentration fields for (a) growth and (b) dissolution

The flux balance condition at the precipitate/matrix interface below must be satisfied.

$$
\left(c^{p}-c^{m}\right) \frac{d R}{d t}=\left.D \frac{\partial c}{\partial r}\right|_{r=R},
$$

where $c^{p}$ is the solute concentration in the precipitate, which is assumed to be a constant independent of $r$ and $t$. The solution of eq. (1) with the boundary conditions eqs. (2) and (3) gives the concentration field $c(r, t)$. The equation for the interface position $R(t)$ is obtained from eq. (6) using the concentration field $c(r, t)$.

For growth the exact solution exists and the invariant field, the invariant size and the linearized gradient approximations are available. For dissolution no exact solution exists although the invariant field and the invariant size approximations are available. The linearized gradient approximation for dissolution is much complicated to use. Three approximations are briefly summarized in the following.

The invariant field approximation is an attempt to simplify the concentration field equation eq. (1) by setting $\partial c / \partial t=0$ and solving the simplified equation. Both the growth and dissolution problems can be solved by this approximation.

The invariant size approximation ${ }^{1)}$ assumes that $R(t)$ is independent of the history of it. The concentration field is given by $c(r, t)=\left[\left(c^{m}-c^{\infty}\right) R / r\right] \operatorname{erfc}[(r-R) / 2 \sqrt{D t}]+$ $c^{\infty}$. Both the growth and dissolution problems can be solved by this approximation.

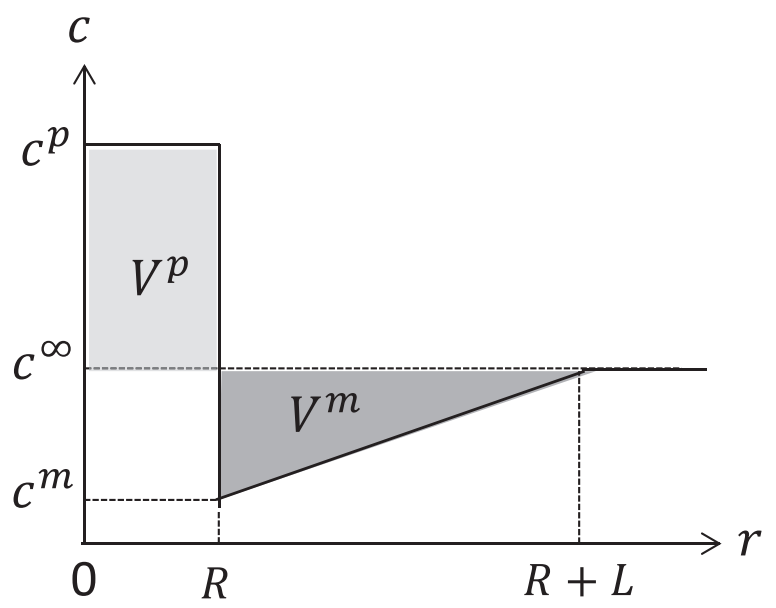

Fig. 2 Linearized gradient approximation for growth. The mass conservation condition, $V^{p}=V^{m}$, is shown.

The linearized gradient approximation ${ }^{1,5)}$ is an attempt to simplify the concentration field by assuming that the concentration gradient is linear in the variable $r$ as shown in Fig. 2. Extent of the linear field $L$ is determined by conservation of mass as shown in the figure. The following equation for the concentration field satisfies the boundary conditions eqs. (2) and (3).

$$
\begin{gathered}
c(r)=\left(c^{m}-c^{\infty}\right)\left(1-\frac{r-R}{L}\right)+c^{\infty}, R \leq r \leq R+L, \\
c(r)=c^{\infty}, R+L \leq r .
\end{gathered}
$$

The linearized gradient approximation gives the solution for growth, but this approximation is not convenient to use practically. The cubic equation ${ }^{9)}$ must be solved to obtain the expression of $L$.

For growth $R$ is proportional to $\sqrt{D t}$ for the exact solution and the approximations. The growth rate constant $\lambda$ is defined bellow.

$$
R(t)=2 \lambda \sqrt{D t} .
$$

$\lambda$ is a function of the supersaturation $\Omega$, which defines the kinetics of growth and dissolution. The supersaturation $\Omega$ for growth is defined as follows.

$$
\Omega=\frac{c^{\infty}-c^{m}}{c^{p}-c^{m}} . \quad(0 \leq \Omega \leq 1)
$$

For dissolution the simple definition for the rate constant $\lambda$ like eq. (8) is not available. The solution for the invariant field approximation for dissolution is the following.

$$
R^{2}=R_{0}^{2}-2 \Omega t .
$$

The supersaturation $\Omega$ for dissolution is defined as follows.

$$
\Omega=\frac{c^{m}-c^{\infty}}{c^{p}-c^{m}} . \quad(0 \leq \Omega)
$$

The solution for the invariant size approximation has not explicit analytical expression. The linearized gradient approximation for dissolution is not available. 


\section{Modification of the Conventional Linearized Gra-} dient Approximation

A new approximation for the growth and dissolution of spherical precipitates is proposed here. It is the modified version of the conventional linearized gradient approximation. 5,6$)$

\subsection{Growth}

Substituting the following equation to eq. (1),

$$
c=\left(c^{m}-c^{\infty}\right) \frac{R}{r} w+c^{\infty},
$$

eq. (1) becomes

$$
\frac{\partial w}{\partial t}=D \frac{\partial^{2} w}{\partial r^{2}} .
$$

The boundary conditions for $w$ are

$$
\begin{array}{ll}
w(R, t)=1, & 0<t, \\
w(\infty, t)=0, & 0<t, \\
w(r, 0)=0, & R \leq r .
\end{array}
$$

Equation (13) is the one dimensional diffusion equation for $w(r, t)$, which is considered as plane flow. Therefore it is reasonable that $w(r, t)$, instead of $c=c(r, t)$, is approximated by the linearized gradient.

$$
\begin{gathered}
w(r)=1-\frac{r-R}{L}, R \leq r \leq R+L, \\
w(r)=0, R+L \leq r .
\end{gathered}
$$

From eqs. (12) and (17), the concentration field is obtained as follows.

$$
\begin{gathered}
c(r)=\left(c^{m}-c^{\infty}\right) \frac{R}{r}\left(1-\frac{r-R}{L}\right)+c^{\infty}, \\
R \leq r \leq R+L, \\
c(r)=c^{\infty}, R+L \leq r .
\end{gathered}
$$

The following relation for $c(r)$ is obtained from the condition of mass conservation shown in Fig. 2.

$$
\begin{aligned}
\frac{4 \pi}{3} R^{3}\left(c^{p}-c^{\infty}\right) & =\int_{R}^{R+L} 4 \pi r^{2}\left(c^{\infty}-c(r)\right) d r \\
& =4 \pi\left(c^{\infty}-c^{m}\right) R \int_{R}^{R+L} r\left(1-\frac{r-R}{L}\right) d r
\end{aligned}
$$

By performing the integral in eq. (19), the following quadratic equation for $L / R$ is obtained using $\Omega$ defined in eq. (9).

$$
\left(\frac{L}{R}\right)^{2}+3\left(\frac{L}{R}\right)-2 \frac{1-\Omega}{\Omega}=0 .
$$

It should be noticed that a cubic equation for $L / R$, which is not easy to solve, ${ }^{9)}$ is obtained for the conventional linearized gradient approximation. The positive solution $\alpha$ of eq. (20) for $L / R$ is calculated using the quadratic formula.

$$
\alpha=\frac{3}{2}\left(-1+\sqrt{1+\frac{8}{9} \cdot \frac{1-\Omega}{\Omega}}\right) .
$$

From eq. (18),

$$
\left.\frac{\partial c}{\partial r}\right|_{r=R}=\left(c^{\infty}-c^{m}\right)\left(1+\frac{R}{L}\right) \frac{1}{R} .
$$

From the flux balance condition eq. (6) and eq. (22), the following equation for $R(t)$ is derived. $\alpha$ is substituted to $L / R$.

$$
\frac{d R}{d t}=\Omega D\left(1+\frac{1}{\alpha}\right) \frac{1}{R},
$$

eq. (23) is integrated with time and $R(t)$ is obtained.

$$
R(t)=\sqrt{2 \Omega D\left(1+\frac{1}{\alpha}\right) t} .
$$

Then, the growth rate constant defined by eq. (8) is

$$
\lambda_{m}=\sqrt{\frac{\Omega}{2}\left(1+\frac{1}{\alpha}\right)} .
$$

Here, subscript $m$ denotes the modified linearized gradient approximation.

\subsection{Dissolution}

The solute concentration field of the modified linearized gradient approximation for dissolution is also described by eq. (18). The mass conservation for the dissolution problem is shown in Fig. 3. The equation, which is different from the growth problem, is obtained by the mass conservation condition.

$$
\begin{aligned}
& \frac{4}{3} \pi\left(R_{0}^{3}-R^{3}\right)\left(c^{p}-c^{\infty}\right) \\
& \quad=\left(c^{m}-c^{\infty}\right) \int_{R}^{R+L} 4 \pi r^{2} \frac{R}{r}\left(1-\frac{r-R}{L}\right) d r .
\end{aligned}
$$

From eq. (26) the following quadratic equation for $L / R$ is obtained.

$$
\left(\frac{L}{R}\right)^{2}+3\left(\frac{L}{R}\right)-2 \frac{1+\Omega}{\Omega}\left(\frac{R_{0}^{3}}{R^{3}}-1\right)=0,
$$

where $\Omega$ is defined by eq. (11). Using the quadratic formula the positive solution $\beta$ of eq. (27) for $L / R$ is obtained.

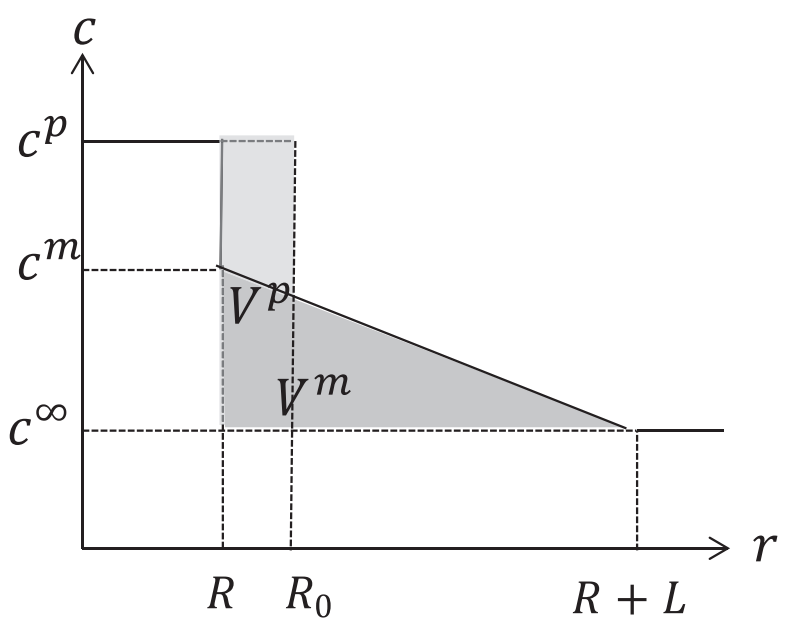

Fig. 3 Linearized gradient approximation for the dissolution. The mass conservation condition, $V^{p}=V^{m}$, is shown. 


$$
\beta=\frac{3}{2}\left(-1+\sqrt{1+\frac{8}{9} \cdot \frac{1+\Omega}{\Omega}\left(\frac{R_{0}^{3}}{R^{3}}-1\right)}\right) .
$$

From eq. (18) the following relation is obtained.

$$
\left.\frac{\partial c}{\partial r}\right|_{r=R}=-\left(c^{\infty}-c^{m}\right)\left(1+\frac{R}{L}\right) \frac{1}{R}
$$

From the flux balance condition eq. (6) and eq. (29), the following equation for $R(t)$ is derived. $\beta$ is substituted to $L / R$.

$$
\frac{d R}{d t}=-\frac{D \Omega}{R}\left(1+\frac{1}{\beta}\right)
$$

Unlike the growth problem, eq. (30) cannot be solved analytically, because $\beta$ includes $R$ in it. It is possible to solve this equation numerically using for example the Runge-Kutta method. ${ }^{10)}$ The numerical solution of the modified linearized gradient approximation is denoted by the modified linearized gradient $(\mathrm{N})$ approximation.

However, solving the equation numerically is inconvenient. If $R^{3}$ in $\beta$ is substituted with the average value $\overline{R^{3}}$ during the precipitate dissolution process, $\beta$ is replaced with a constant $\langle\beta\rangle$. Then eq. (30) is solved analytically to obtain $R(t) . R(t)$ has the following expression.

$$
R^{2}=R_{0}^{2}\left(1-t / t^{d i s}\right),
$$

where, $t^{d i s}$ is the dissolution time for precipitates. The average of $R^{3}$ is calculated by the following equation without knowing the expression of $t^{d i s}$.

$$
\overline{R^{3}}=R_{0}^{3} \int_{0}^{t^{d i s}}\left(1-t / t^{d i s}\right)^{\frac{3}{2}} d t / t^{d i s}=\frac{2}{5} R_{0}^{3} .
$$

Substituting $5 / 2$ to $R_{0}^{3} / \overline{R^{3}}$ in eq. (28), $\langle\beta\rangle$ is as follows.

$$
\langle\beta\rangle=\frac{3}{2}\left(-1+\sqrt{1+\frac{4}{3} \cdot \frac{1+\Omega}{\Omega}}\right) .
$$

From eq. (33) and the boundary condition eq. (5b), eq. (30) can be solved and the following expression for $R^{2}$ is obtained.

$$
R^{2}=R_{0}^{2}-2 \Omega D\left(1+\frac{2}{3} \cdot \frac{1}{-1+\sqrt{1+\frac{4}{3} \cdot \frac{1+\Omega}{\Omega}}}\right) t
$$

The analytical expression obtained from the approximation of the modified linearized gradient approximation is denoted by the modified linearized gradient (A) approximation.

\section{Results and Discussion}

Tables 1 and 2 summarize the various solutions for the growth and dissolution of spherical precipitates including the present modified linearized approximation together with their limiting forms for $\Omega \ll 1$.

\subsection{Growth}

\begin{tabular}{|c|c|}
\hline Solution & $\begin{array}{c}\text { Growth Rate Contants, } \\
R=2 \lambda \sqrt{D t} \\
\Omega=\left(c^{\infty}-c^{m}\right) /\left(c^{p}-c^{m}\right)(0 \leq \Omega \leq 1)\end{array}$ \\
\hline Exact solution & $\begin{array}{r}2 \lambda_{e}^{2} \times\left[1-\sqrt{\pi} \lambda_{e} \exp \left(\lambda_{e}^{2}\right) \operatorname{erfc}\left(\lambda_{e}\right)\right]=\Omega \\
\lambda_{e} \approx \lambda_{f}, \text { for } \Omega \ll 1\end{array}$ \\
\hline $\begin{array}{l}\text { Invariant field } \\
\text { approximation }\end{array}$ & $\lambda_{f}=\sqrt{\frac{\Omega}{2}}$ \\
\hline $\begin{array}{l}\text { Invariant size } \\
\text { approximation }\end{array}$ & $\lambda_{s}=\frac{\Omega}{2 \sqrt{\pi}}+\sqrt{\frac{\Omega}{2}\left(1+\frac{\Omega}{2 \pi}\right)}$ \\
\hline & $\lambda_{s} \approx \lambda_{f}$, for $\Omega \ll 1$ \\
\hline \multirow[t]{2}{*}{$\begin{array}{l}\text { Linerized gradient } \\
\text { approximation }\end{array}$} & $\begin{array}{l}\lambda_{l}=\sqrt{\frac{\Omega}{2(A+B-4 / 3)^{\prime}}} \\
\text { where }\end{array}$ \\
\hline & $\lambda_{l} \approx 2^{-\frac{1}{6}}(\Omega / 2)^{\frac{2}{3}}$, for $\Omega \ll 1$ \\
\hline $\begin{array}{l}\text { Modified } \\
\text { linearized gradient } \\
\text { approximation } \\
\text { (present work) }\end{array}$ & $\lambda_{m}=\sqrt{\frac{\Omega}{2}}\left(1+\frac{2}{3} \cdot \frac{1}{-1+\sqrt{1+\frac{8}{9} \cdot \frac{1-\Omega}{\Omega}}}\right)$ \\
\hline & $\lambda_{m} \approx \sqrt{\frac{\Omega}{2}}\left(1+\sqrt{\frac{\Omega}{2}}\right)$, for $\Omega \ll 1$ \\
\hline
\end{tabular}

In Fig. 4, the growth rate constants $\lambda$ 's for the spherical precipitate are calculated as a function of the supersaturation $\Omega$ using the exact solution, the invariant field, the invariant size, the conventional linear gradient and the modified linearized gradient approximations. The approximations are
Table 1 Solutions for growth of spherical precipitate.

Table 2 Solutions for dissolution of spherical precipitate.

\begin{tabular}{lc}
\hline \multicolumn{1}{c}{ Solution } & $\begin{array}{c}\text { Relation between } Z \text { and } \tau, \\
\text { where } Z \equiv R / R_{0}, \tau \equiv D t / R_{0}^{2} .\end{array}$ \\
$\Omega=\left(c^{m}-c^{\infty}\right) /\left(c^{p}-c^{m}\right)(0 \leq \Omega)$
\end{tabular}




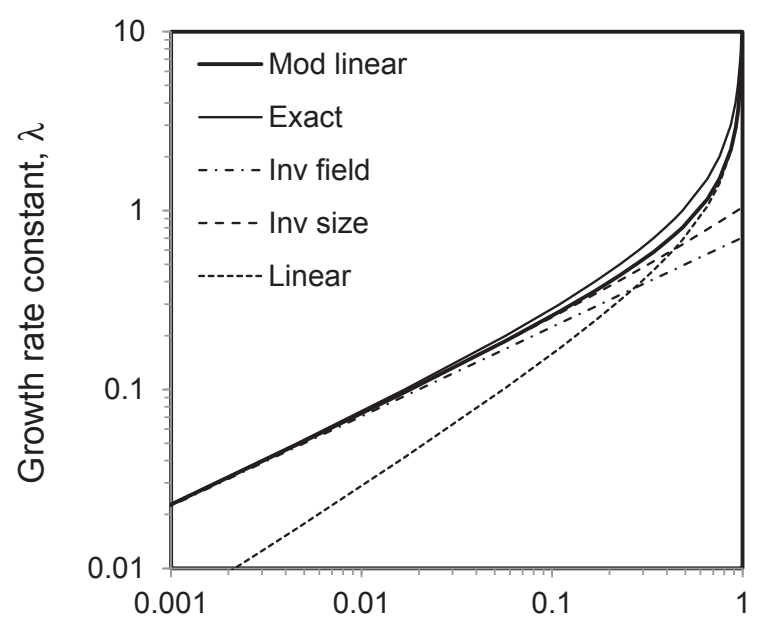

Supersaturation, $\Omega$

Fig. 4 Growth rate constant as a function of the supersaturation $\Omega$ for various solutions.

compared with the exact solution. The exact solution always has the largest $\lambda$. The invariant field and the invariant size approximations deviate from the exact solution at large $\Omega$. The modified linearized gradient approximation is the best among all the approximations considered here. The conventional linearized gradient approximation deviates largely from the exact solution for the small value of $\Omega$. Only the modified linearized gradient approximation can approximate the exact solution for the whole range of $\Omega(0<\Omega<1)$.

In Fig. 5 the concentration fields for the modified linearized gradient approximate solution for growth are plotted. The concentration fields for the exact and the conventional linearized gradient approximate solutions are also plotted. In the figure $\left(c-c^{\infty}\right) /\left(c^{\infty}-c^{m}\right)$ is the reduced concentration field and $r / 2 \sqrt{D t}$ is the dimensionless reduced radial coordinate. It is shown that the concentration field for the modified linearized gradient approximation is close to the one for exact solution at small $\Omega$.

\subsection{Dissolution}

The dissolution problem is difficult to study, as no exact solution exist contrary to the growth problem. Therefore, it is hard to evaluate the validity of the approximations. However, it seems to be reasonable to use the modified linearized gradient approximation as a reference for evaluating the validity of the approximations for dissolution, because it is a good approximation for the growth problem. In the present discussion, therefore, the numerical solution of the modified linearized gradient approximation is considered as a reference.

Dissolution rate cannot be easily defined, so the simple way to study the approximate solutions for dissolution is to show the relation between precipitate radius and time. In the following the radius and the time are normalized by $R_{0}$ and $D / R_{0}^{2}$ respectively. They are denoted by $Z$ and $\tau$.

From the analytical expressions in Table 2 it is clear that $Z^{2}$ for the invariant field approximation is always larger than that for the invariant size and the modified linearized gradient (A) approximations. As shown in Table 2, the modified
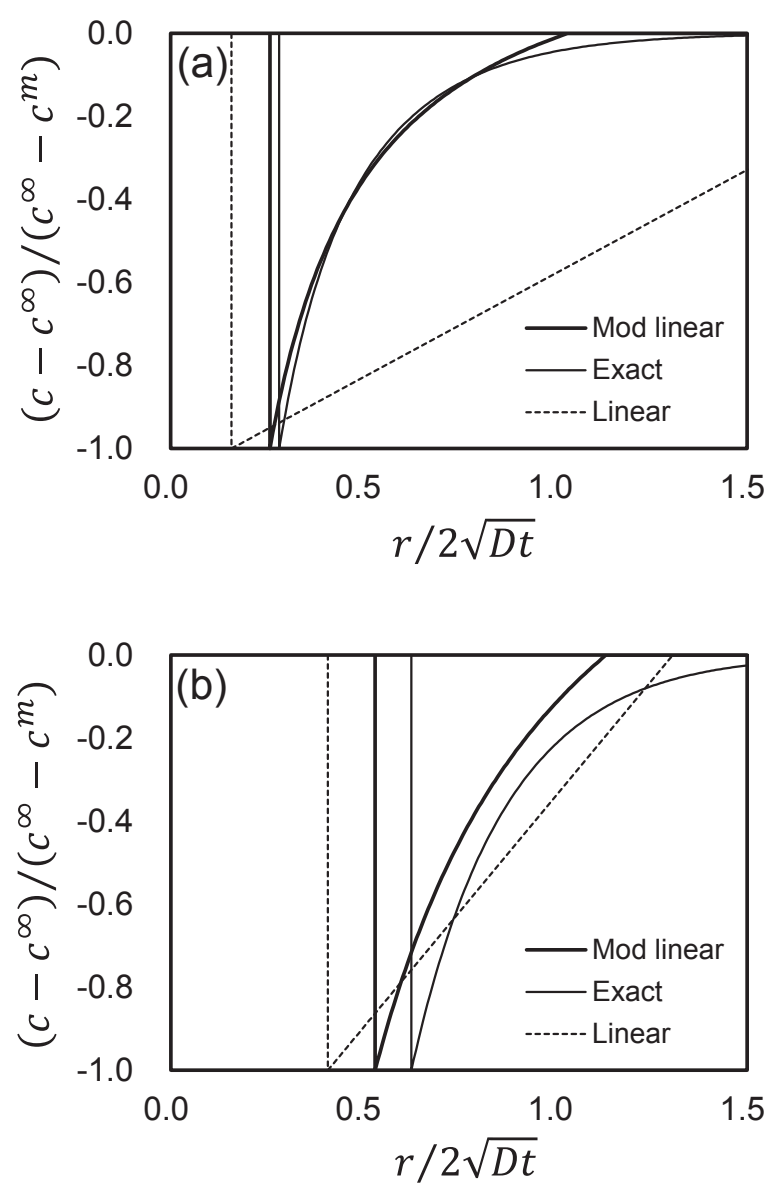

Fig. 5 The concentration fields for the modified linearized gradient approximation for growth, (a) $\Omega=0.1$, (b) $\Omega=0.3$. $\left(c-c^{\infty}\right) /$ $\left(c^{\infty}-c^{m}\right)$ is the reduced concentration field and $r / 2 \sqrt{D t}$ is the dimensionless reduced radial coordinate.

linearized gradient (A) and the invariant size approximate solutions reduce to the invariant field approximate solution at $\Omega \ll 1$ as follows.

$$
Z^{2}=1-\Omega \tau, \text { for } \Omega \ll 1 .
$$

Figure 6 shows the normalized precipitate radius $Z$ vs. the normalized time $\tau$ for the modified (N) and (A) linearized gradient, the invariant field and the invariant size approximations. In the figure two cases of $\Omega=0.05$ and $\Omega=0.2$ are plotted. $Z^{2}$ for the modified linearized gradient $(\mathrm{N})$ approximation is calculated numerically. The explicit analytical form for $Z^{2}$ of the invariant size approximation cannot be obtained, thus $Z^{2}$ of that approximation is obtained numerically. The figure shows that $Z^{2}$ for the modified linearized gradient $(\mathrm{N})$ and $(\mathrm{A})$ approximations are always between those for the invariant field and the invariant size approximations. The modified linearized $(\mathrm{N})$ approximation is close to the invariant size approximation, which is suggested to use by Aaron et al. ${ }^{1)}$ The difference between (N) and (A) approximations is not large. The modified (A) linearized gradient approximation should be probably used for dissolution. The modified linearized gradient (A) approximation is more convenient than the modified linearized gradient $(\mathrm{N})$ approximation.

The normalized dissolution time $\tau^{\text {dis }}$, which is the time normalized by $R_{0}^{2} / D$ to disappear for the precipitate whose 


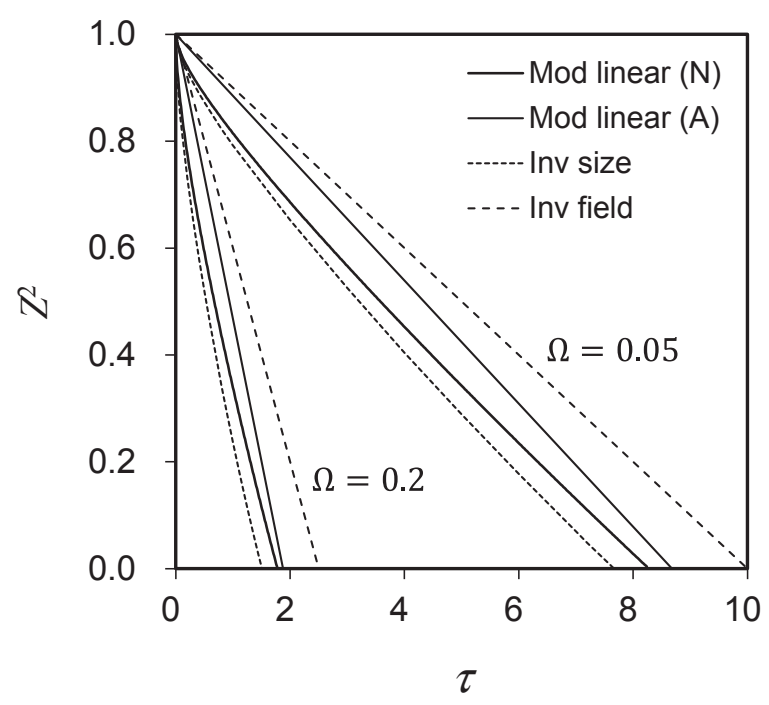

Fig. 6 Dissolution of spherical precipitates according to the modified linearized gradient $(\mathrm{N})$, the modified linearized gradient $(\mathrm{A})$, the invariant field and the invariant size approximations for $\Omega=0.2$ and $\Omega=0.05$. The radius and time are normalized by $R_{0}$ and $R_{0}^{2} / D$, respectively.

Table 3 Analytic expressions for dissolution time.

\begin{tabular}{ll}
\hline \multicolumn{1}{c}{ Solution } & \multicolumn{1}{c}{ Dissolution time normalized by $R_{0}^{2} / D$} \\
\hline $\begin{array}{l}\text { Invariant field } \\
\text { approximation }\end{array}$ & $\tau_{f}^{d i s}=\frac{1}{2 \Omega}$ \\
$\begin{array}{l}\text { Invariant size } \\
\text { approximation }\end{array}$ & $\begin{array}{l}\tau_{s}^{d i s}=\frac{1}{2 \Omega} \exp \left(-\frac{2 \Omega}{\sqrt{2 \pi \Omega-\Omega^{2}}} \operatorname{arccot}\left(\frac{\Omega}{\sqrt{2 \pi \Omega-\Omega^{2}}}\right)\right), \\
\text { for } 0<\Omega<2 \pi\end{array}$ \\
& $\tau_{s}^{d i s}=\frac{1}{2 \Omega}\left(\frac{\Omega-\sqrt{\Omega^{2}-2 \pi \Omega}}{\Omega+\sqrt{\Omega^{2}-2 \pi \Omega}}\right)^{\Omega / \sqrt{\Omega^{2}-2 \pi \Omega}}$, for $2 \pi \leq \Omega$ \\
$\begin{array}{l}\text { Modified } \\
\text { lA } \text { apprized gradient } \\
\text { (present work) }\end{array}$ & $\tau_{m(A)}^{d i s}=\frac{1}{2 \Omega}\left(1+\frac{2}{3} \cdot \frac{1}{-1+\sqrt{1+\frac{4}{3} \cdot \frac{1+\Omega}{\Omega}}}\right)^{-1}$ \\
\hline
\end{tabular}

initial radius is $R_{0}$, is calculated as a function of $\Omega$ for each approximation. The analytical forms of $\tau^{d i s}$ are given in Table 3 for the invariant field, the invariant size and the modified linearized gradient (A) approximations. For the modified linearized gradient $(\mathrm{N})$ approximation $\tau^{\text {dis }}$ is calculated numerically. Figure 7 shows $\tau^{\text {dis }}$ vs. $\Omega$ for the various approximations. $\tau^{d i s}$ 's for the modified linearized gradient $(\mathrm{N})$ and $(\mathrm{A})$ approximations are between the invariant field and the invariant size approximations. $\tau^{\text {dis' }} \mathrm{S}$ for the modified linearized gradient $(\mathrm{N})$ and (A) approximations are very close for the wide range of $\Omega$.

\section{Summary}

A new approximation to the exact solutions of the growth and dissolution of the spherical precipitates have been proposed. The present approximation is based on the linearized gradient approximation. The present approximation for growth is much improved compared with the conventional linearized approximation. The dissolution problem is more difficult to treat than the growth problem. It has been shown that the present modified linearized

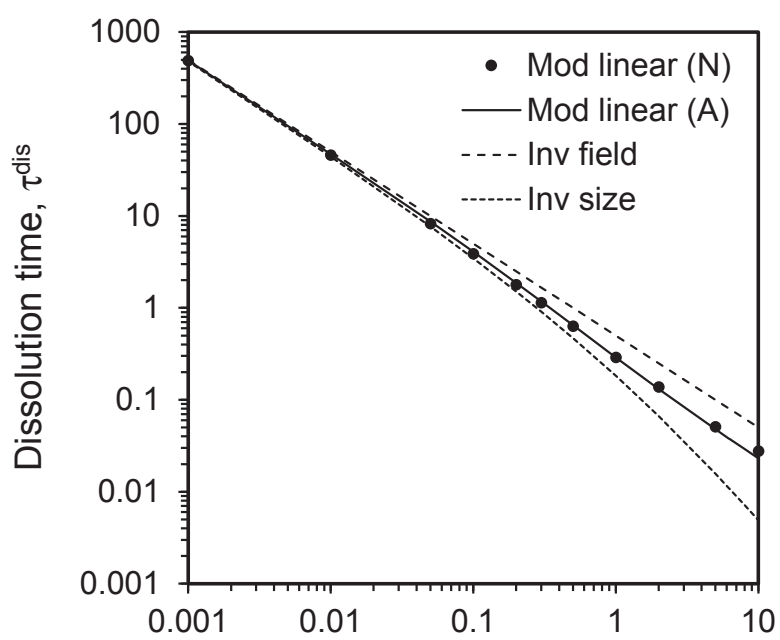

Supersaturation, $\Omega$

Fig. 7 Dissolution time for the modified linearized gradient $(\mathrm{N})$, the modified linearized gradient (A), the invariant field and the invariant size approximations. The time is normalized by $R_{0}^{2} / D$.

gradient approximation is also applicable to the dissolution problem. The present approximation is convenient and useful in the dissolution problem, where the conventional linearized gradient approximation and the exact solution does not exist.

\section{Appendix}

\section{Reduced Concentration Field of the Exact Solution for Growth}

The reduced concentration field $u$ of the exact solution is expressed by the reduced radial coordinate $x$ as follows. ${ }^{1)}$

$$
u=-\frac{\lambda}{e^{-\lambda^{2}}-\lambda \sqrt{\pi} \operatorname{erfc}(\lambda)}\left(\frac{1}{x} e^{-x^{2}}-\sqrt{\pi} \operatorname{erfc}(x)\right),
$$

where $u=\left(c-c^{\infty}\right) /\left(c^{\infty}-c^{m}\right)$ and $x=r / 2 \sqrt{D t} . \quad \lambda$ is obtained for given $\Omega$ solving the transcendental equation for the exact solution in Table 1. The reduced position of the interface $X$ is calculated as follows.

$$
X=R / 2 \sqrt{D t}=\lambda
$$

\section{REFERENCES}

1) H.B. Aaron, D. Fainstein and G.R. Kotler: J. Appl. Phys. 41 (1970) 4404-4410.

2) H.I. Aaronson, M. Enomoto and J.K. Lee: Mechanism of Diffusional Phase Transformations in Metals and Alloys, (CRC Press, Boca Raton, 2010) pp. 253-264, 297-303.

3) J. Crank: The Mathematics of Diffusion, (Oxford University Press, New York, 1975) pp. 308-310.

4) S.R. Coriell and R.L. Parker: J. Appl. Phys. 36 (1965) 632-637.

5) C. Zener: J. Appl. Phys. 20 (1949) 950-953.

6) M. Enomoto and H.I. Aaronson: J. Appl. Phys. 51 (1980) 818-819.

7) H. Chen and S. van der Zwagg: J. Mater. Sci. 46 (2011) 1328-1336.

8) L.C. Brown: J. Appl. Phys. 47 (1976) 449-458.

9) Handbook of Mathematical Functions with Formulas, Graphs and Mathematical Tables, ed. by M. Abramowitz and I.A. Stegun, (U. S. Gov. Printing Office, Washington, D.C, 1966) p. 17.

10) W.H. Press, B.P. Flannery, S.A. Teukolsky and W.T. Vetterling: Numerical Recipes, (Cambridge University Press, Cambridge, 1986) pp. $550-554$. 